\title{
From a PMT-based to a SiPM-based PET system: a study to define matched acquisition/reconstruction parameters and NEMA performance of the Biograph Vision 450
}

Thomas Carlier $^{1,2^{*}}$ (D) Ludovic Ferrer ${ }^{2,3}$, Maurizio Conti ${ }^{4}$, Caroline Bodet-Milin ${ }^{1,2}$, Caroline Rousseau ${ }^{2,3}$, Yanic Bercier ${ }^{4}$, Bernard Bendriem ${ }^{4}$ and Françoise Kraeber-Bodéré ${ }^{1,2}$

\footnotetext{
* Correspondence: thomas.carlier@ chu-nantes.fr

${ }^{1}$ Nuclear Medicine Department University Hospital of Nantes, Place Alexis Ricordeau, Nantes, France 2Université de Nantes, CNRS, INSE RM, CRCINA, Nantes, France Full list of author information is available at the end of the article
}

\begin{abstract}
Background: The purpose of this work was to propose an approach based on noise measurement to adapt present clinical acquisition and reconstruction parameters adapted to a PMT-based system (Biograph $\mathrm{mCT}$ ) to a SiPM-based system (Biograph Vision 450) sharing identical geometrical properties. The NEMA performance (NEMA) of the recently released Biograph Vision 450 PET/CT (Vision) was also derived.

Methods: All measurements were conducted on Vision and Biograph $\mathrm{mCT}$ with TrueV (mCT). A full NEMA-based performance was derived for Vision only. The adaptation of acquisition and reconstruction parameters from $\mathrm{mCT}$ to Vision was done using the NEMA image quality phantom. The noise level reached using $\mathrm{mCT}$ was set as the reference value for six different numbers of net true coincidences. The noise level computed using Vision was matched to the reference noise level (within $0.01 \%$ ) using a different reconstruction set-up to determine the potential reduction of count numbers for the same noise level.

Results: Vision sensitivity was $9.1 \mathrm{kcps} / \mathrm{MBq}$ for a timing resolution of 213 ps at 5.3 $\mathrm{kBq} / \mathrm{mL}$. The NEMA-based CR for the $10-\mathrm{mm}$ sphere was better than $75 \%$ regardless the reconstruction set-up studied. The $\mathrm{mCT}$ reference noise properties could be achieved using Vision with a scan time reduction (STR) of 1.34 with four iterations and a $440 \times 440$ matrix size (or STR $=1.89$ with a $220 \times 220$ matrix size) together with a 3D CR improvement of 53\% for the $10-\mathrm{mm}$ sphere ( $24 \%$ using $220 \times 220$ ).

Conclusion: The Vision exhibited improved NEMA performances compared to $\mathrm{mCT}$. Using the proposed approach, the time acquisition could be divided by almost two, while keeping the same noise properties as that of $\mathrm{MCT}$ with a marked improvement of contrast recovery.
\end{abstract}

Keywords: Vision 450, NEMA, Noise, Optimization
(อ) The Author(s). 2020 Open Access This article is licensed under a Creative Commons Attribution 4.0 International License, which permits use, sharing, adaptation, distribution and reproduction in any medium or format, as long as you give appropriate credit to the original author(s) and the source, provide a link to the Creative Commons licence, and indicate if changes were made. The images or other third party material in this article are included in the article's Creative Commons licence, unless indicated otherwise in a credit line to the material. If material is not included in the article's Creative Commons licence and your intended use is not permitted by statutory regulation or exceeds the permitted use, you will need to obtain permission directly from the copyright holder. To view a copy of this licence, visit http://creativecommons.org/licenses/by/4.0/. 


\section{Background}

The interest for using silicon photomultipliers (SiPMs) in clinical PET imaging was initially motivated by the known incompatibility of photomultiplier tubes with intense magnetic fields when considering the combination of a PET detector within an MRI system [1]. More specifically, the first released clinical simultaneous PET/MR Biograph mMR (Siemens Healthineers) used avalanche photodiodes (APDs) as photodetectors [2], which prevented the use of time-of-flight (TOF) information due to the low timing resolution. This difficulty was subsequently addressed by the use of SiPMs, which allow a higher gain (at least $10^{3}$ ) and a faster rise time (by roughly a factor of three) than APDs [3]. The first TOF PET/MR based on SiPMs technology was released by General Electric (GE Healthcare) with a timing resolution of about 400 ps [4].

Since then, there has been a growing interest to also use SiPMs as photodetectors in PET/CT systems since it paves the way towards a more compact detector, together with an improved timing resolution and a finer decoding scheme of the crystal signal. Indeed, it was shown that highest timing resolution has many advantages in PET/CT among improved signal-to-noise ratio, faster convergence of iterative algorithms, lesser sensitivity to inconsistent or missing data, better imaging properties in cases of very low statistics, and better detectability through the reduction of voxel size [5-7]. The first clinical system with a timing resolution of below $250 \mathrm{ps}$ was recently released by Siemens with the Biograph Vision PET/CT. Two versions are currently available (600 and 450), which differ in the axial field of view (FOV): $26.3 \mathrm{~cm}$ for version 600 and $19.7 \mathrm{~cm}$ for version 450. The NEMA performance [8] was published for version 600 [9] but not for version 450. The timing resolution for the Vision 600 was measured to be between 210 and 215 ps across a wide range of count rates.

The purpose of this study was, firstly, to derive the NEMA performance of the Vision $450 \mathrm{PET} / \mathrm{CT}$ and, secondly, to make a direct comparison of it to the similar (in terms of geometry) mCT with the extended FOV. This study demonstrates the possible improvement achievable when an PMT-based system is replaced with a SiPM-based with the same geometrical properties and the benefits of using an enhanced TOF resolution in the clinical workflow. Two clinical examples are also presented to illustrate the possible improvements in routine clinical practice.

\section{Methods}

\section{Biograph vision $450 \mathrm{PET} / \mathrm{CT}$}

The PET component of the Vision 450 is very similar to that of the version 600 . It contains 38 blocks per ring for six rings along the axial FOV. Each block is subdivided into $4 \times 2$ mini-blocks (four mini-blocks in tangential position for two mini blocks in the axial position), that each contain an array of $5 \times 5$ LSO crystals of $3.2 \times 3.2 \times 20 \mathrm{~mm}^{3}$. The mini block is coupled to an array of $16 \times 16 \mathrm{~mm}^{2}$ SiPMs. Table 1 summarizes the main properties of the Vision 450.

\section{NEMA measurements}

All measurements were performed following the NEMA procedure including spatial resolution, sensitivity, scatter fraction, noise equivalent count rate (NECR) and 
Table 1 Technical specifications of the PET component for Vision 450 (Vision 600 within brackets) and Biograph $\mathrm{mCT}$

\begin{tabular}{lll}
\hline Characteristics & Vision 450 (Vision 600) & Biograph mCT \\
\hline Crystal dimension & $3.2 \times 3.2 \times 20 \mathrm{~mm}^{3}$ & $4 \times 4 \times 20 \mathrm{~mm}^{3}$ \\
Number of crystals per mini block & $5 \times 5$ & $\mathrm{NA}$ \\
Number of mini blocks per block & $4 \times 2$ & $\mathrm{NA}$ \\
Number of blocks per ring & 38 & 48 \\
Number of blocks in axial FOV & $6(8)$ & 4 \\
Axial FOV (cm) & $19.7(26.3)$ & 21.6 \\
Image planes & $119(159)$ & 109 \\
Plane spacing (mm) & 1.65 & 2 \\
Bed overlap for step and shoot mode (number of slices) & $57(79)$ & 47 \\
Maximum ring difference & $59(79)$ & 49 \\
Span & 17 & 11 \\
Coincidence time window (ns) & 4.7 & 4.1 \\
Energy window (keV) & $435-585$ & $435-650$ \\
\hline
\end{tabular}

accuracy, timing resolution, image quality, and co-registration accuracy. The different experiments were analyzed using the software provided by the manufacturer.

\section{Spatial resolution}

Because of the expected high spatial resolution of the Vision, $\mathrm{a}{ }^{22} \mathrm{Na}$ point source (352 $\mathrm{kBq}$ ) with a dimension (diameter $0.25 \mathrm{~mm}$ ) suitable to the crystal size was used for all measurements. The source was located in the FOV at given transaxial position $(x, y)$ where $\mathrm{x}$ and $\mathrm{y}$, expressed in centimeters, were at the following positions in a given $\mathrm{z}$ plane: $(0,1),(0,10)$, and $(0,20)$ at a $z$-position of $1 / 8$ axial FOV and $1 / 2$ axial FOV. The precise position of the source was controlled through a pre-localization step using a specific source L-fixture developed by the manufacturer to ensure that the source was within $\pm 2 \mathrm{~mm}$ for the $(\mathrm{x}, \mathrm{y})$ positions and $\pm 0.25 \mathrm{~mm}$ for the $\mathrm{z}$ positions. Two million net true coincidences (defined as prompt minus random coincidences) were collected.

Data were reconstructed using a Fourier rebinning combined with a filtered back projection without attenuation, scatter corrections, and using a ramp filter. The voxel size was $0.83 \times 0.83 \times 0.83 \mathrm{~mm}^{3}$ (matrix size $880 \times 880 \times 237$ ).

\section{Sensitivity}

A 700-mm long polyethylene tube (inside diameter $1 \mathrm{~mm}$; outside diameter $3 \mathrm{~mm}$ ) was filled with $4.9 \mathrm{MBq}$ of ${ }^{18} \mathrm{~F}$ at the start of data acquisition. The source was placed inside the sleeves and positioned at the center and at a $10-\mathrm{cm}$ radial offset. Five data sets corresponding to five specific wall thicknesses were acquired for $300 \mathrm{~s}$ each.

\section{Scatter fraction and NECR}

A cylinder of polyethylene (700-mm long and a diameter of $200 \mathrm{~mm}$ ) was used. A line source (inside diameter $3 \mathrm{~mm}$, outside diameter $4.8 \mathrm{~mm}$ ) was inserted axially into the cylinder at a radial position of $45 \mathrm{~mm}$ from the phantom center. The initial activity in the line source was $1156 \mathrm{MBq}$ of ${ }^{18} \mathrm{~F}$. Thirty-five frames of $240 \mathrm{~s}$ were acquired in 11.3 
h. The random coincidences were accounted for using the delayed coincidence technique.

\section{Timing resolution}

The timing resolution was calculated based on the experiment involving the measurement of scatter fraction and NECR. It was estimated as a function of concentration activity following the method proposed by Wang et al. [10].

\section{Image quality}

The torso-shaped IEC phantom with six coplanar spheres (internal diameters of 10, 13, $17,22,28$ and $37 \mathrm{~mm}$ ) was used to evaluate image quality. A central cylindrical insert simulating lung tissue was added to the IEC phantom. The background was filled with a concentration of $5.4 \mathrm{kBq} / \mathrm{mL}$ of ${ }^{18} \mathrm{~F}$-FDG while the four smallest spheres were filled so that the concentration ratio between the spheres and the background was 4:1 (the two largest spheres were filled with non-radioactive water). Acquisition was performed with the spheres' center aligned with the axial center of the FOV. The phantom used for the scatter fraction and NECR was placed axially near the IEC phantom to simulate activity outside the FOV. The activity in the line source of this phantom was $153 \mathrm{MBq}$ at the start of the acquisition. The acquisition time was set to $240 \mathrm{~s}$.

Data were reconstructed using the TOF 3D ordinary Poisson ordered subset expectation maximization (3D OP-OSEM) algorithm with point spread function (PSF) recovery and TOF. Two matrix sizes were considered: $220 \times 220$ (voxel size $3.2 \times 3.2 \times 1.65 \mathrm{~mm}^{3}$ ) and $440 \times 440$ (voxel size $\left.1.65 \times 1.65 \times 1.65 \mathrm{~mm}^{3}\right)$. The reconstruction parameters were close to those used in routine clinical practice: four iterations and five subsets without post-filtering. For comparison purposes, data were also reconstructed following the reconstruction parameters matching those used in the seminal work of van Sluis et al. using the Biograph Vision 600 [9] for image quality assessment. The percentage contrast recovery (CR) for each sphere, the percent background variability, the residual errors for attenuation, and scatter corrections were then computed as specified by the NEMA guidelines [8].

\section{Co-registration accuracy}

A small vial filled with $35 \mathrm{MBq}$ of ${ }^{18} \mathrm{~F}$ and a CT contrast agent $(240 \mathrm{mg} / \mathrm{mL})$ was used for this measurement. The vial was positioned at three transaxial coordinates: $(0,1),(0$, $20)$, and $(20,0)$ centimeters. A total weight of $115 \mathrm{~kg}$ was positioned on the table, and the co-registration accuracy was evaluated at two axial positions $(5 \mathrm{~cm}$ and $100 \mathrm{~cm}$ from the tip of the pallet). A CT scan followed by a 3-min PET acquisition was performed for the six positions considered. PET images were reconstructed using 3D OP-OSEM (10 iterations, 5 subsets, no post-filtering, $440 \times 440$ matrix size). The coregistration error was subsequently calculated using the software provided by the manufacturer as well as the maximum ratios defined in the NEMA guidelines [8].

\section{Image quality comparison between vision 450 and $\mathrm{mCT}$}

A direct comparison between the Vision and the analog-based $\mathrm{mCT}$ with the extended axial FOV [11] was conducted. This comparison will help guide how to sort patients between the two systems and how to maintain consistency in longitudinal studies 
involving both systems. For this purpose, the IEC NEMA phantom with a sphere-tobackground contrast of 4:1 was first acquired on the Vision for $240 \mathrm{~s}$ and immediately after on the $\mathrm{mCT}$ for $250 \mathrm{~s}$ to account for radioactive decay.

The noise level (described hereafter) typically observed using the $\mathrm{mCT}$ (with a standard clinical acquisition and reconstruction protocol) was set as the reference value for different numbers of net true coincidences corresponding to acquisition times of 4,3 , $2,1.5,1$, and $0.5 \mathrm{~min}$. The same number of net true coincidences was chosen for both systems (within 0.01\%). The noise level computed using the Vision was matched to the reference noise level (described below) using different reconstruction set-ups (matrix size and reconstruction parameters) to determine the potential reduction of count numbers for the same noise level. Indeed, a similar noise level could be reached with a lower number of counts thanks to the improved time resolution of the Vision. The noise level was computed using the image roughness (IR) as described by Tong et al. [12] based on a single $27-\mathrm{cm}^{3}$-spherical region-of-interest (ROI) so that the same computation could be used with patient data. IR was defined following:

$$
I R=\frac{\sqrt{\frac{1}{N} \sum_{i \in R O I}\left(v_{i}-\bar{m}\right)^{2}}}{\bar{m}}
$$

where $N$ is the number of voxels in the ROI, $v_{\mathrm{i}}$ the value of voxel $\mathrm{i}$, and $\bar{m}$ the mean of voxels in the ROI. The image roughness measures the pixel-to-pixel variation and is closely related to the visual perception of noise using a single image. This metric was preferred over background variability (BV) defined in the NEMA standards because BV captures more region-to-region variability, which is better adapted to quantify the variance of background measurement. As noise is not the only parameter relevant in this context, the CR for the hot and cold spheres in 3D considering the entire sphere volume (as opposed to the 2D evaluation used in the NEMA evaluation computed for a 2D cross-section of each sphere) was also computed for each final set-up so that a comparison of contrast could also be achieved. Each voxel intersected by the theoretical surface of the sphere was considered in the computation. The reference reconstruction parameters $(\mathrm{mCT})$ for the 3D OP-OSEM+TOF + PSF used were three iterations and 21 subsets using a matrix size of $200 \times 200$ (voxel size $4 \times 4 \times 2 \mathrm{~mm}^{3}$ ). A post-filtering was not applied to ease the interpretation of the results. Additionally, the CR (calculated in 3D) for each sphere size was also derived as a function of IR.

Two clinical cases were also considered to qualitatively illustrate the possible benefits derived from the proposed methodology. As a true whole-body comparison is nearly impossible with ${ }^{18}$ F-FDG given the time difference between the two imaging sessions, two patients were selected with an identical mass, size, and time between injection and PET imaging. The first patient was a 73-year-old $(56 \mathrm{~kg}, 1.56 \mathrm{~m})$ woman evaluated for a breast cancer (injected activity, $173 \mathrm{MBq}$ of ${ }^{18} \mathrm{~F}$-FDG; time delay between injection and imaging, $63 \mathrm{~min}$; system used, Biograph $\mathrm{mCT}$; reconstruction parameters, 3D OPOSEM+PSF + TOF with three iterations, twenty-one subsets, and no post-filtering). The second patient was a 78-year-old $(56 \mathrm{~kg}, 1.57 \mathrm{~m})$ woman evaluated for a recurrence of follicular lymphoma (injected activity, $173 \mathrm{MBq}$ of ${ }^{18} \mathrm{~F}$-FDG; time delay between injection and imaging, $60 \mathrm{~min}$; system used, Vision 450; reconstruction parameters, 3D OP-OSEM+PSF + TOF with four iterations, five subsets, and no post-filtering). Vision 
450 data were reconstructed by adapting the acquisition time to what was found by using the phantom experiment. Image roughness was calculated in a homogeneous region of the right lobe of the liver for each reconstruction.

The second clinical case referred to a patient treated with ${ }^{90} \mathrm{Y}$-microspheres $(2251 \mathrm{MBq}$ of Therapshere ${ }^{\circ}$ for a segment VII hepatocellular carcinoma. The radioembolization was for the management of a local recurrence including several satellite nodules. The acquisition time was $30 \mathrm{~min}$ for $\mathrm{mCT}$ ( 1 bed step) and Vision ( 2 beds step of $15 \mathrm{~min}$ ). The reconstruction parameters were OP-OSEM+PSF + TOF, 3 iterations, 21 subsets, and no post-filtering for mCT $(200 \times 200$ matrix size $)$ and OP-OSEM+PSF + TOF, 4 iterations, 5 subsets, and no post-filtering for Vision as recently suggested in this specific case [13]. The time delay between injection and acquisition was $45.3 \mathrm{~h}$ for Vision and $46.1 \mathrm{~h}$ for $\mathrm{mCT}$.

\section{Results}

\section{NEMA measurements}

\section{Spatial resolution}

Table 2 gives the spatial resolution for each source position in terms of FWHM and full width at tenth maximum (FWTM). Results were averaged over two independent measurements.

\section{Sensitivity}

The sensitivity was $9.1 \mathrm{kcps} / \mathrm{MBq}$ at the center of the FOV while it was $8.9 \mathrm{kcps} / \mathrm{MBq}$ at $10 \mathrm{~cm}$ radial distance from the center of the FOV (Fig. 1a).

\section{Scatter fraction and NECR}

Figure $1 \mathrm{~b}$ shows the plots of scatter, true, random, prompt coincidences rate, and NECR as a function of activity concentration. Scatter fraction is shown in Fig. 2.

The peak NECR was $160 \mathrm{kcps}$ at $29.3 \mathrm{kBq} / \mathrm{mL}$, and the peak true rate was $694 \mathrm{kcps}$ at $51.9 \mathrm{kBq} / \mathrm{mL}$. The scatter fraction was $37.5 \%$ at peak NECR and $36.2 \%$ at low activity.

Table 2 Spatial resolution performance

\begin{tabular}{llll}
\hline Parameter & & & \\
\hline Spatial resolution & Position $(\mathrm{cm})$ & FWHM $(\mathrm{mm})$ & FWTM (mm) \\
Radial & 1 & 3.5 & 6.7 \\
& 10 & 4.5 & 8.2 \\
& 20 & 5.8 & 10.0 \\
Tangential & 1 & 3.8 & 7.5 \\
& 10 & 4.0 & 7.4 \\
& 20 & 3.6 & 6.7 \\
Axial & 1 & 3.5 & 6.9 \\
& 10 & 4.2 & 8.3 \\
& 20 & 4.2 & 8.9 \\
\hline
\end{tabular}

Source: Na22 source, $0.25-\mathrm{mm}$ diameter

Reconstruction: Fourier rebinning combined with a filtered back projection without attenuation, scatter corrections , and no post-filtering. The voxel size was $0.83 \times 0.83 \times 0.83 \mathrm{~mm}^{3}$ (matrix size $880 \times 880 \times 237$ ) 

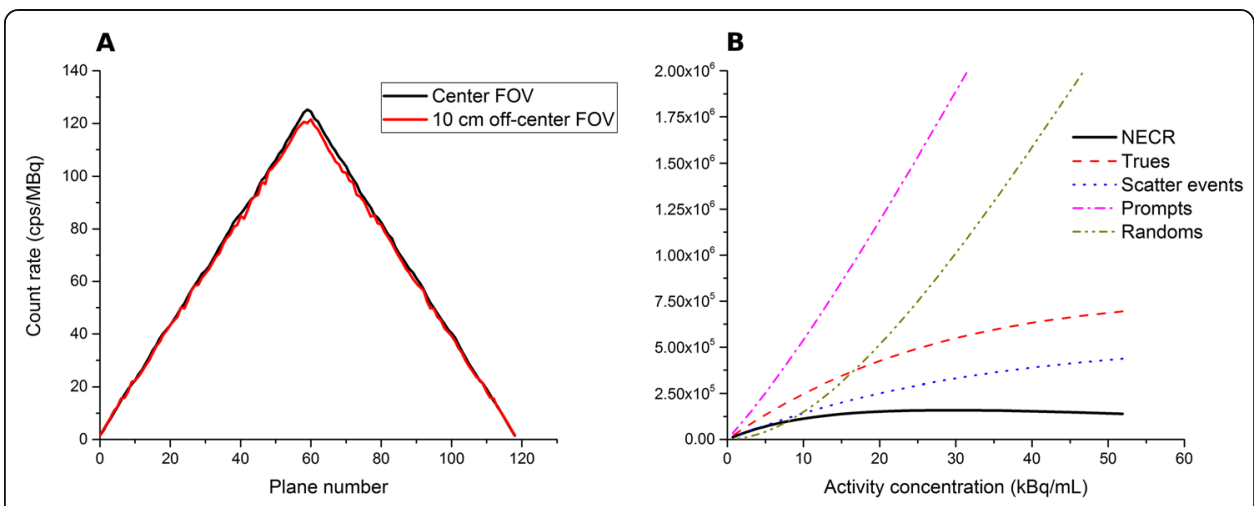

Fig. 1 a Sensitivity profile at the center and $10 \mathrm{~cm}$ off-center of the FOV. b NECR and count rates for prompt, true, scatter, and random events

\section{Timing resolution}

Figure 2 illustrates the dependence of time resolution on activity concentration. The timing resolution was $213 \mathrm{ps}$ at $5.3 \mathrm{kBq} / \mathrm{mL}$ and varied at most $3 \%$ over the activity range studied.

\section{Image quality and co-registration error}

Table 3 summarizes the results for the 4:1 contrast ratio and for the two matrix sizes studied $(220 \times 220$ and $440 \times 440)$. The maximum co-registration error was found to be $1.12 \mathrm{~mm}$. All maximum ratios for both PET and CT were less than 0.3 (range [0.160.23] for PET and [0.05-0.18] for CT).

Image quality comparison between vision 450 and $\mathrm{mCT}$

Figure 3 shows the $3 \mathrm{D}$ contrast recovery as a function of IR for each sphere size for the two systems using the $440 \times 440$ (Vision) and the $200 \times 200(\mathrm{mCT})$ matrix size using

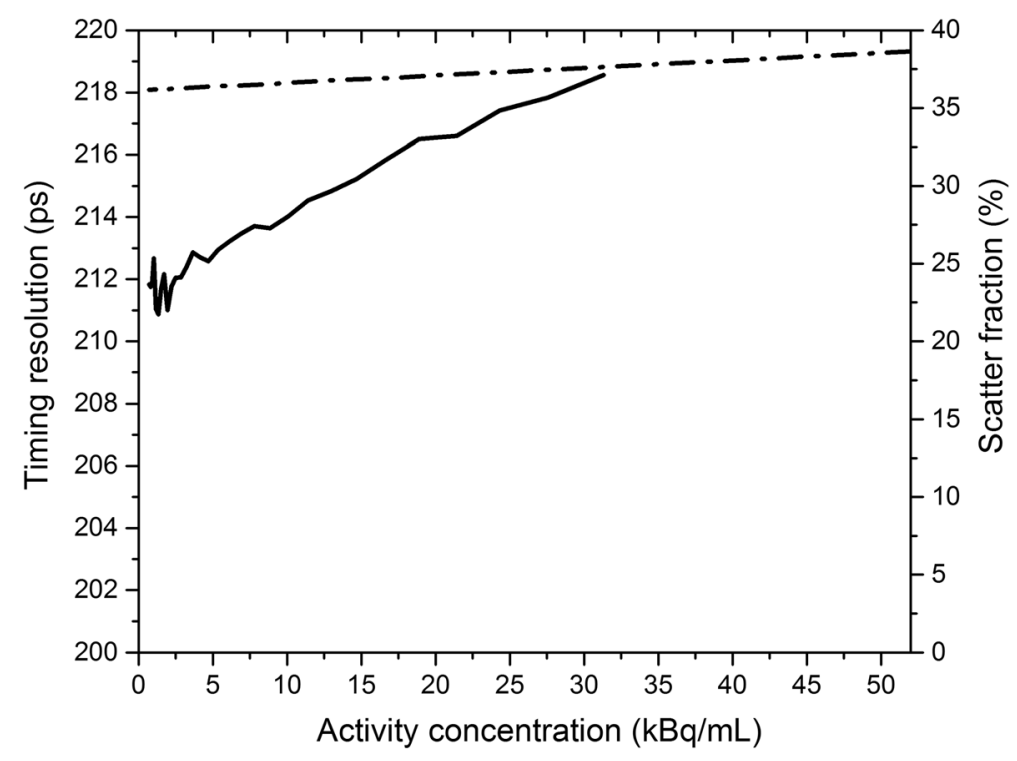

Fig. 2 Scatter fraction (dashed-dotted line) and time resolution (solid line) for the activity range studied 
Table 3 Image quality NEMA results for the 4:1 contrast ratio (3D OP-OSEM+PSF + TOF, 4 iterations, 5 subsets, no post-filtering)

\begin{tabular}{|c|c|c|c|c|}
\hline \multirow[b]{2}{*}{ Matrix size } & \multicolumn{2}{|c|}{ Contrast recovery (\%) } & \multicolumn{2}{|c|}{ Background variability (\%) } \\
\hline & $220 \times 220$ & $440 \times 440$ & $220 \times 220$ & $440 \times 440$ \\
\hline \multicolumn{5}{|l|}{ Sphere diameter $(\mathrm{mm})$} \\
\hline 10 & 75.4 & $83.3(94.4)$ & 5.3 & $6.3(9.5)$ \\
\hline 13 & 78.4 & $82.4(88.0)$ & 4.4 & $5.0(7.3)$ \\
\hline 17 & 91.0 & $93.2(96.5)$ & 3.4 & $3.7(5.2)$ \\
\hline 22 & 96.3 & $98.7(101.8)$ & 2.7 & $2.7(3.8)$ \\
\hline 28 & 76.7 & $77.6(84.6)$ & 2.3 & $2.3(3.1)$ \\
\hline 37 & 83.2 & $84.1(89.9)$ & 1.9 & $1.9(2.5)$ \\
\hline Average lung residual (\%) & 5.5 & $5.5(3.2)$ & & \\
\hline
\end{tabular}

Results obtained using 8 iterations, 5 subsets, and no post-filtering are reported within brackets. These values could be directly compared to those reported in Table 3 in [9], for the 10,13, 17, and 22-mm spheres

the same number of net trues $\left(63.4 \times 10^{6}\right.$ coincidences). The comparison to $\mathrm{mCT}$ using the $400 \times 400$ matrix size is provided in supplementary material 1 . Figure 4 plots the IR as a function of the number of net trues for the two systems. Both high and low resolution Vision reconstructions show lower noise than the reference low resolution $\mathrm{mCT}$ reconstruction, for all iteration numbers considered. As expected, increasing iteration number and lower pixel size resulted in higher noise levels. For comparison purposes, data reconstructed using the $400 \times 400$ matrix size are presented in supplementary material 2 .

Given the noise reduction offered by excellent time resolution in the Vision, a reduction of counts (equivalently: acquisition time) can occur. Using the IR as the measure of noise, the possible time reduction factor was computed by fixing the IR of the reference curve and then finding the number of net trues required to reach these IR reference values when reconstructing data using the Vision with different reconstruction

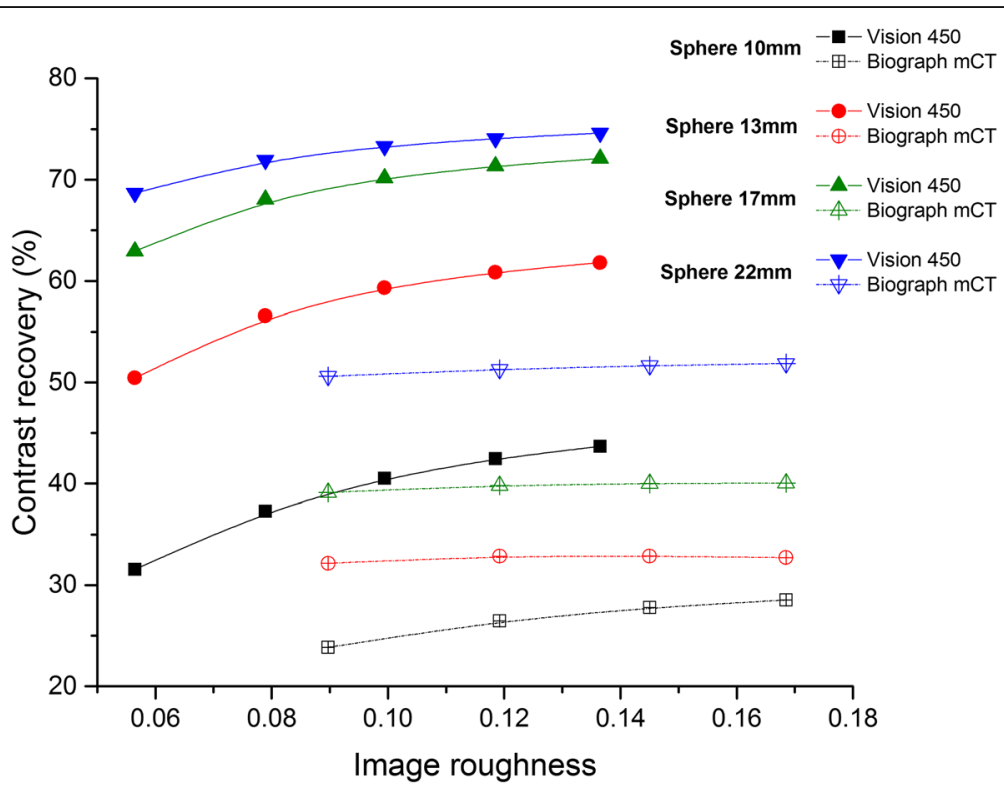

Fig. 3 3D contrast recovery computed for Vision (solid line) and $\mathrm{mCT}$ (dotted line). Each point is for a specific number of iterations. Datasets from the two scanners have the same number of net trues 

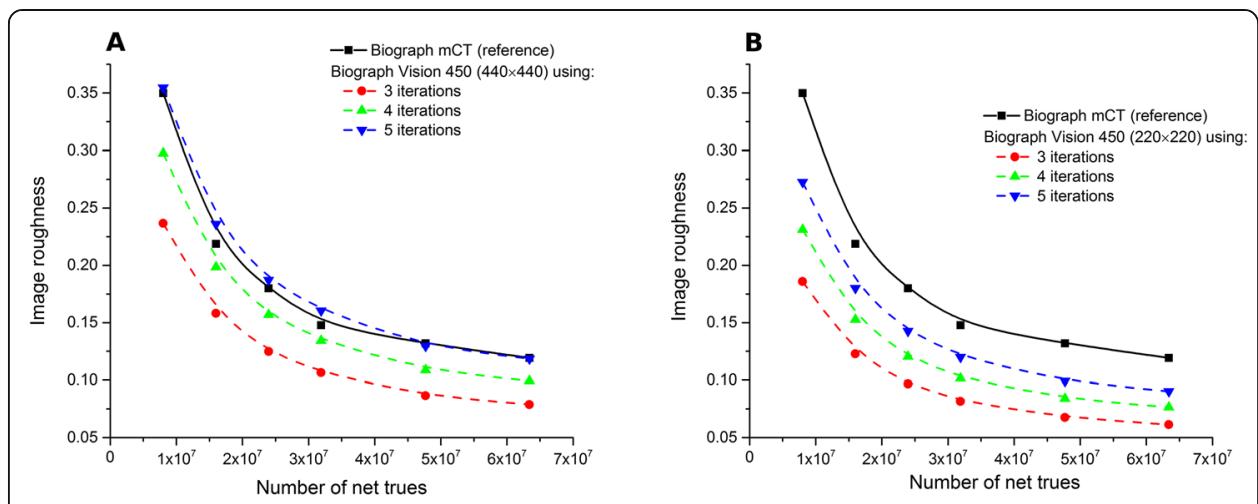

Fig. 4 Image roughness as a function of the number of net trues. Data reconstructed from the $\mathrm{mCT}$ (set as the reference) using 3 iterations, 21 subsets, and no post-filtering (matrix size $200 \times 200$ ). Vision data were reconstructed using 5 subsets, no post-filtering, and between 3 to 5 iterations. a $440 \times 440$ and b $220 \times$ 220 matrix size. Data were fitted using a bi-exponential to ease the visual interpretation

parameters (limited to 3, 4, and 5 iterations and 5 subsets). Each curve was arbitrarily fitted with a bi-exponential function to increase the precision (or number of points) of the reduction factor computation and ease the visualization. Figure 5 shows the results for the $440 \times 440$ and the $220 \times 220$ matrix size. The mean reduction factors for each case are given in Tables 4 and 5 . They are computed only for a number of net trues less than $35 \times 10^{6}$, as this is mainly the condition encountered in clinical routine practice [14]. The gain in terms of contrast recovery was also reported. This last one was derived for each number of iterations from the reconstruction with the highest number of net trues, as the CR was not supposed to change with the number of net trues. This assessment was conducted using the $200 \times 200$ matrix size from the $\mathrm{mCT}$ as reference (additional results when considering the $400 \times 400$ matrix size are presented in supplementary material 3).

Figure 6 illustrates the effect of reducing the time acquisition using the IEC phantom when considering the image reconstructed with the $\mathrm{mCT}$ as the reference. In this case, the target IR was set to 0.15 (equivalent to a time acquisition of $120 \mathrm{~s}$ for the $\mathrm{mCT}$ ). The reduction factor was defined as the reduction in time that would produce an image

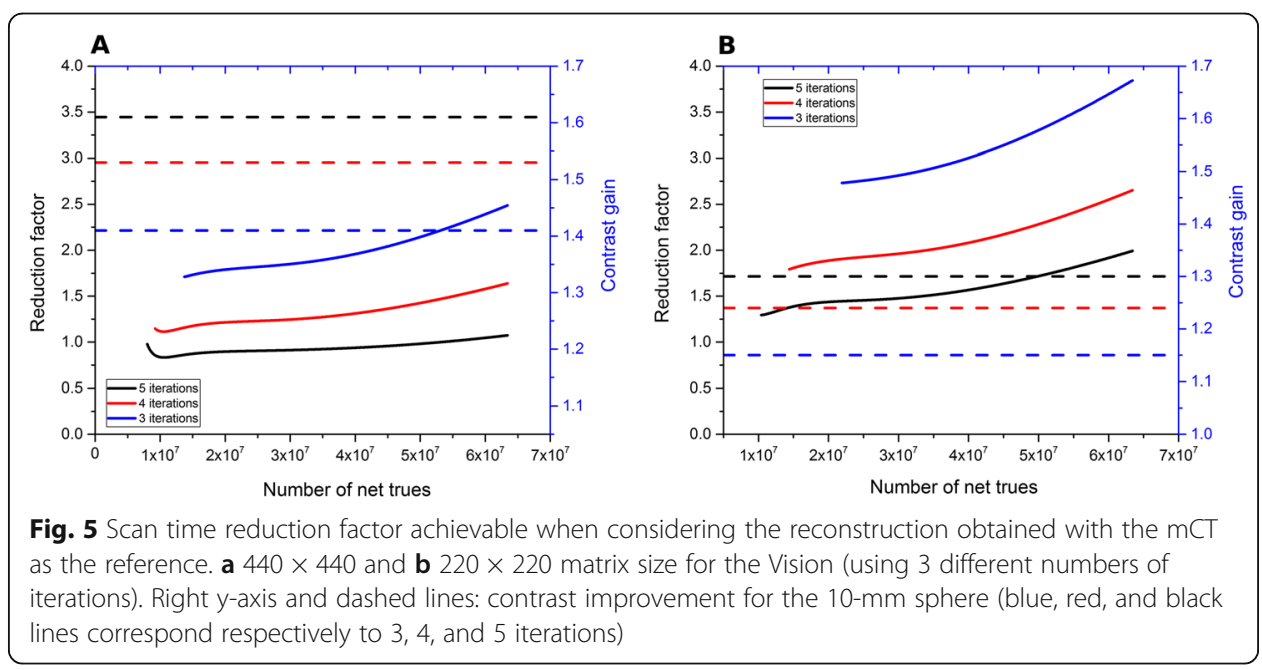


Table 4 Scan time reduction factor and CR gain for different numbers of iterations (Vision with $440 \times 440$ matrix size) when compared to the reference values of $\mathrm{mCT}$

\begin{tabular}{llllllll}
\hline \multirow{2}{*}{$\begin{array}{l}\text { Number of } \\
\text { iterations }\end{array}$} & Mean reduction & \multicolumn{7}{l}{ Contrast recovery gain (\%) } \\
\cline { 2 - 8 } & factor & sphere & sphere & $\begin{array}{l}17-\mathrm{mm} \\
\text { sphere }\end{array}$ & $\begin{array}{l}22-\mathrm{mm} \\
\text { sphere }\end{array}$ & $\begin{array}{l}28-\mathrm{mm} \\
\text { sphere }\end{array}$ & $\begin{array}{l}37-\mathrm{mm} \\
\text { sphere }\end{array}$ \\
\hline 3 & 1.79 & $+41 \%$ & $+72 \%$ & $+71 \%$ & $+40 \%$ & $+14 \%$ & $+9 \%$ \\
4 & 1.34 & $+53 \%$ & $+81 \%$ & $+76 \%$ & $+43 \%$ & $+20 \%$ & $+14 \%$ \\
5 & 0.95 & $+61 \%$ & $+85 \%$ & $+79 \%$ & $+44 \%$ & $+25 \%$ & $+17 \%$ \\
\hline
\end{tabular}

matching the target IR $=0.15$. The reconstructed images for Vision using the $440 \times$ 440 and the $220 \times 220$ matrix size (four iterations and five subsets for both) are presented and corresponded to a reduction time factor of, respectively, 1.34 (acquisition time $89 \mathrm{~s}$ ) and 1.89 (acquisition time $63 \mathrm{~s}$ ). The clinical case using ${ }^{18}$ F-FDG for two patients injected with the same activity and sharing the same mass and size is presented in Fig. 7. The reduction factor derived previously was applied to reconstructed data acquired with the Vision. Given that the reference acquisition time per step using the Biograph mCT was $120 \mathrm{~s}$, data were reconstructed using $220 \times 220$ matrix size and $63 \mathrm{~s}$ per step (which represented a scan time reduction of 1.89) and $89 \mathrm{~s}$ per step (which represented a scan time reduction of 1.34) using $440 \times 440$ matrix size. The IR computed in the right lobe liver was almost identical for all reconstruction: 0.176 for the patient imaged with $\mathrm{mCT}$ ( $120 \mathrm{~s}$ per step), 0.178 and 0.183 for the patient imaged with Vision (respectively $220 \times 220$ and $440 \times 440$ matrix size). The marked improvement of IR when using a matched time acquisition between $\mathrm{mCT}$ and Vision is also highlighted as expected for either the $220 \times 220$ or $440 \times 440$ matrix size. Conversely, a true comparison (that is, same patient imaged using Vision 450 and immediately after, within $5 \mathrm{~min}$, using between Biograph $\mathrm{mCT}$ ) is presented in Fig. 8 for the patient injected with ${ }^{90} \mathrm{Y}$-microspheres. In this specific case, the reconstruction set-up was the one suggested recently in [13].

\section{Discussion}

NEMA performances

NEMA performances of the Vision 450 were compatible to those published, for the Vision 600, in the work of van Sluis et al. [9]. Specifically, spatial resolution measurements were in line, within a few percent, with those reported for the Vision 600 . Sensitivity was around $9.1 \mathrm{kcps} / \mathrm{MBq}$ at the center of the FOV, which was $45 \%$ lower than that of the Vision $600(16.4 \mathrm{kcps} / \mathrm{MBq})$ and $6 \%$ lower than the value reported for

Table 5 Reduction factor and CR gain for different numbers of iterations (Vision with $220 \times 220$ matrix size) when compared to the reference values of $\mathrm{mCT}$

\begin{tabular}{|c|c|c|c|c|c|c|c|}
\hline \multirow[b]{2}{*}{$\begin{array}{l}\text { Number of } \\
\text { iterations }\end{array}$} & \multirow[b]{2}{*}{$\begin{array}{l}\text { Mean reduction } \\
\text { factor }\end{array}$} & \multicolumn{6}{|c|}{ Contrast recovery gain (\%) } \\
\hline & & $\begin{array}{l}\text { 10-mm } \\
\text { sphere }\end{array}$ & $\begin{array}{l}\text { 13-mm } \\
\text { sphere }\end{array}$ & $\begin{array}{l}\text { 17-mm } \\
\text { sphere }\end{array}$ & $\begin{array}{l}\text { 22-mm } \\
\text { sphere }\end{array}$ & $\begin{array}{l}\text { 28-mm } \\
\text { sphere }\end{array}$ & $\begin{array}{l}\text { 37-mm } \\
\text { sphere }\end{array}$ \\
\hline 3 & 2.77 & $+15 \%$ & $+37 \%$ & $+40 \%$ & $+14 \%$ & $+1 \%$ & $+0 \%$ \\
\hline 4 & 1.89 & $+24 \%$ & $+43 \%$ & $+44 \%$ & $+16 \%$ & $+7 \%$ & $+4 \%$ \\
\hline 5 & 1.44 & $+30 \%$ & $+46 \%$ & $+46 \%$ & $+17 \%$ & $+10 \%$ & $+6 \%$ \\
\hline
\end{tabular}



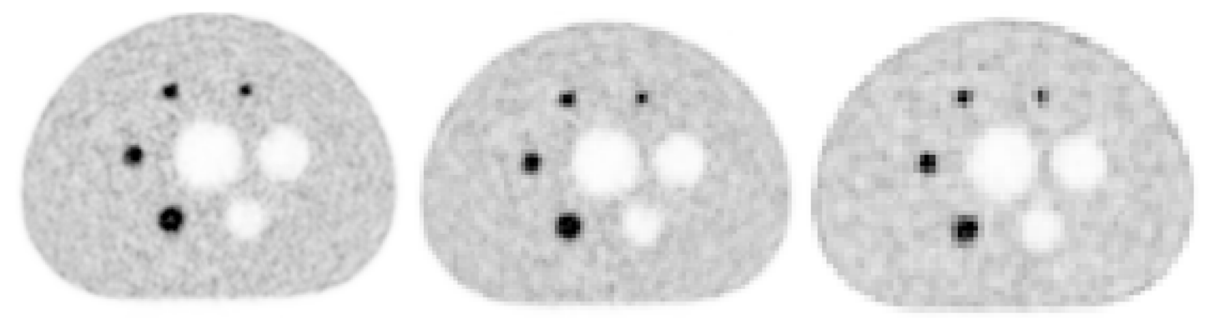

Fig. 6 Reconstructed images of the IEC phantom with the same image roughness $(I R=0.15)$. The reference was for the mCT using 3 iterations, 21 subsets, no post-filtering, $200 \times 200$ matrix size, and $3.2 \times 10^{7}$ net trues (equivalent to a time acquisition of 120 s). Left: Vision using 4 iterations, 5 subsets, no post-filtering, $440 \times 440$ matrix size, and $2.4 \times 10^{7}$ net trues (reduction factor $=1.34$, time acquisition $=89 \mathrm{~s}$ ); middle: Vision using 4 iterations, 5 subsets, no post-filtering, $220 \times 220$ matrix size, and $1.7 \times 10^{7}$ net trues (reduction factor $=1.89$, time acquisition $=63 \mathrm{~s}$ ); right: $\mathrm{mCT}$ (reference). Gray scale level is identical for all images. Contrast improvement for the different spheres, as compared to the reference, is listed in Tables 4 and 5.

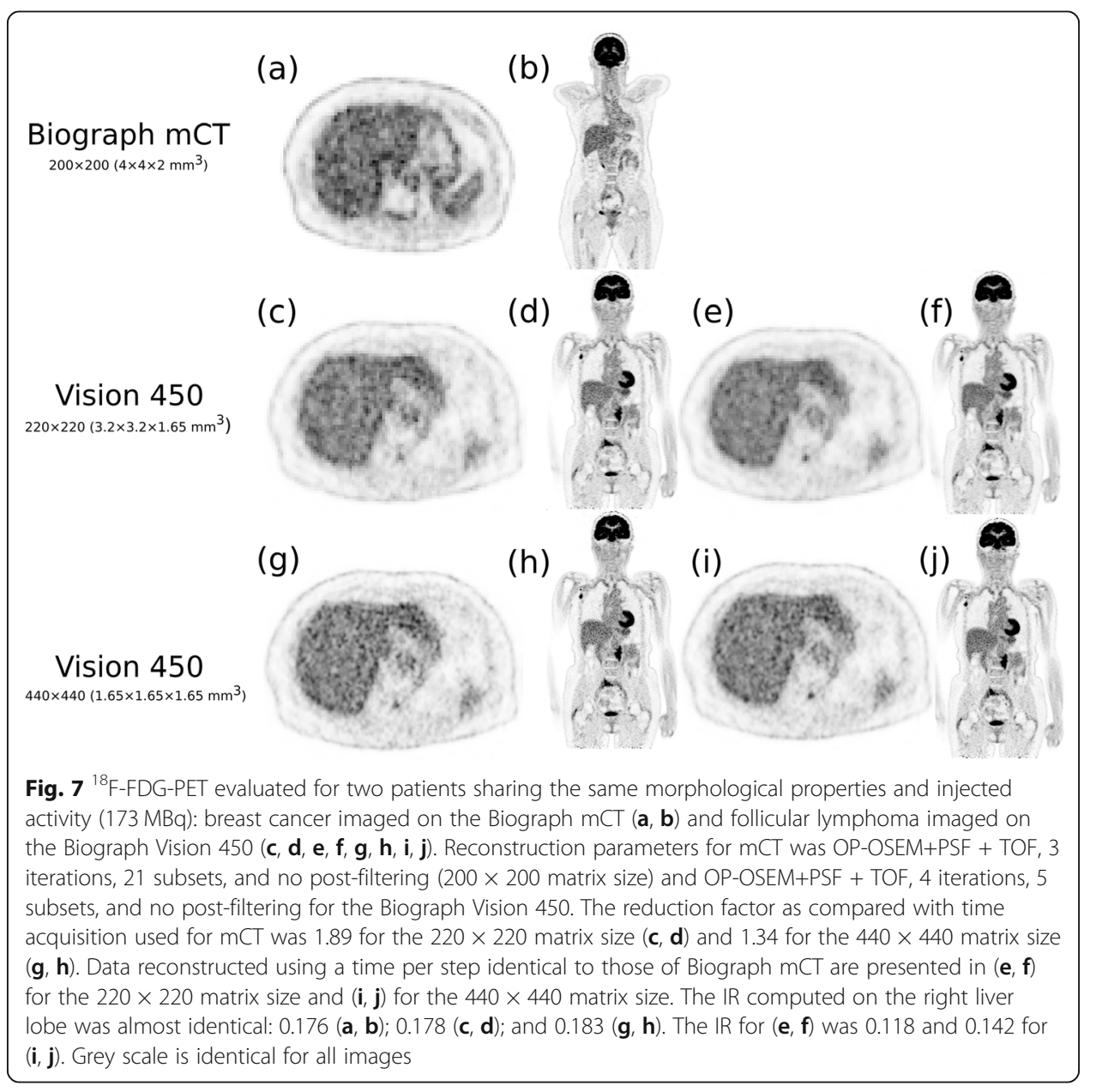




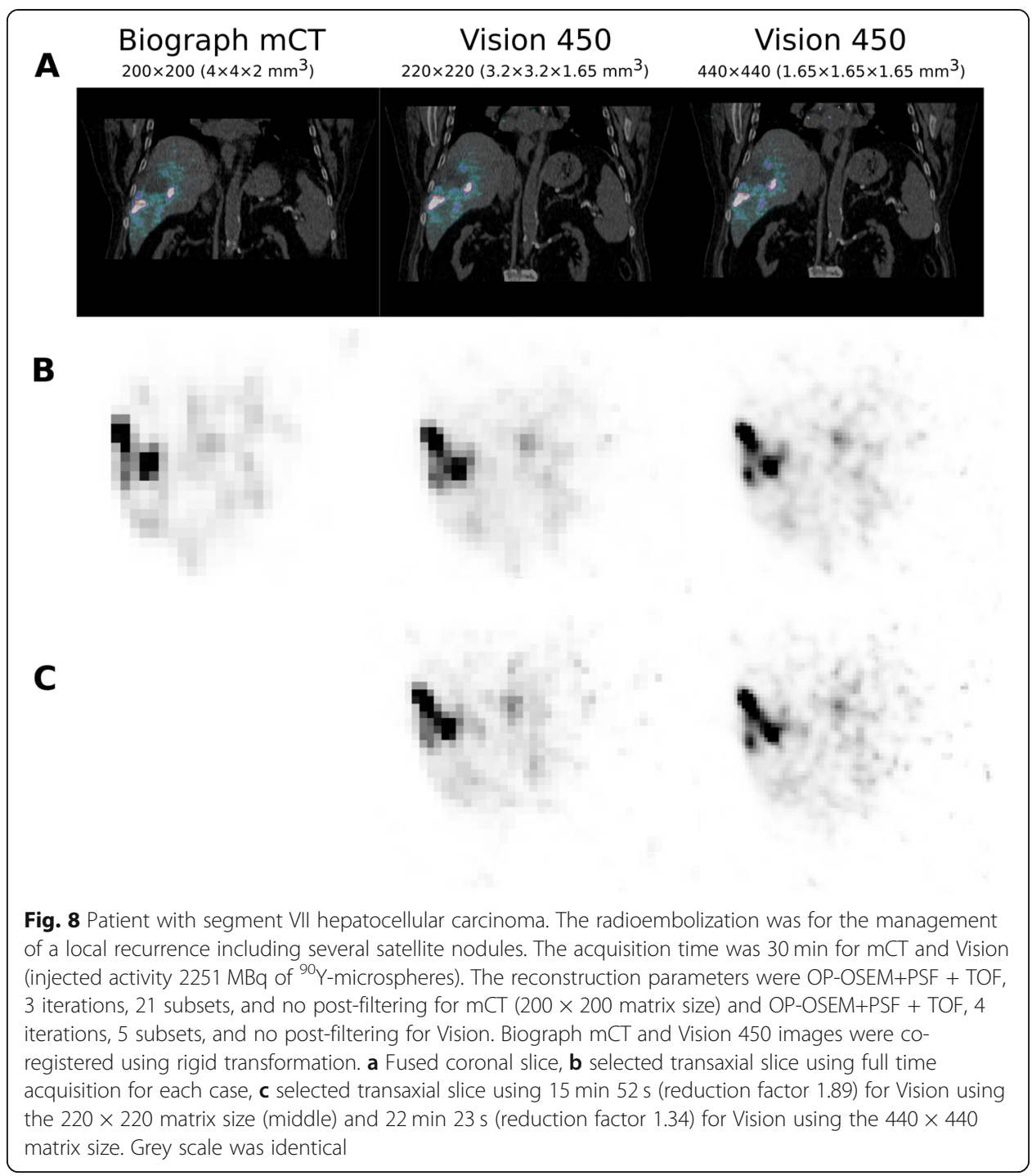

the $\mathrm{mCT}(9.7 \mathrm{kcps} / \mathrm{MBq})$ [11]. This was most likely due to the difference in the axial FOV between the different systems: $26.3 \mathrm{~cm}$ and $21.8 \mathrm{~cm}$ for the Vision 600 and $\mathrm{mCT}$, respectively, and $19.7 \mathrm{~cm}$ for the Vision 450 . It is interesting to note that the experimental ratio of sensitivity between Vision 600 and Vision 450 is well in accordance with the ratio calculated using the model proposed by Eriksson and colleagues [15] (experimental 1.80 vs model 1.84 ).

Accordingly, the peak NECR was found to be $48 \%$ lower than the value reported for the Vision 600 (11\% for the $\mathrm{mCT}$ ) while the scatter fraction remained the same $37.5 \%$ for Vision 450 vs $37 \%$ for Vision 600) at low activity. The time resolution reported with the Vision 600 [9] was confirmed in this study as $213 \mathrm{ps}$ at $5 \mathrm{kBq} / \mathrm{mL}$, very close to the $210 \mathrm{ps}$ of the previous publication. The image quality measurement as proposed by the NEMA standard depended heavily on the reconstruction algorithm used. The 2D contrast recovery was $75 \%$ and $83 \%$ for the $220 \times 220$ and $440 \times 440$ matrices, respectively, which were higher than the $52 \%$ and $65 \%$ reported in [11]. The reconstruction parameters used were identical to those used in [11]. This highlights the benefit of the improved time resolution acquired with the smaller crystal size of the Vision 450 as 
compared to those embedded in the Biograph mCT. Additionally, the 2D contrast recovery for the 10 -mm-diameter sphere was found to be roughly the same $(94.4 \%$ vs 93.1\%) as results obtained with the Vision 600 [9]. Figure 3 showed that the marked 3D contrast recovery improved together with a concomitant significant noise reduction, as measured by the image roughness. This was a direct consequence of a better time resolution, as described previously [7].

\section{Clinical benefits achievable in routine practice}

The installation of a SiPM-based system in a department often raises several questions about the choice of acquisition and reconstruction parameters to be used, especially when the system replaces a PMT-based system with an identical geometry and hence a very similar sensitivity. In this work, a simple method was proposed to give an idea of what the reduction factor could be, given a targeted reference noise level defined using standard parameters of a PMT-based system. Specifically, it was shown that a noise level set using standard reconstruction parameters of the $\mathrm{mCT}$ could be obtained with a reduction factor of 1.34 (four iterations, five subsets, and a $440 \times 440$ image matrix) or of 1.89 (four iterations, five subsets, and a $220 \times 220$ image matrix). Each reduction factor resulted in significant 3D contrast recovery improvement. For example, the 10$\mathrm{mm}$-diameter sphere benefited from an increase of $+53 \%$ (respectively, $+24 \%$ ) when using the $440 \times 440$ matrix and, respectively, the $220 \times 220$ matrix (Tables 4 and 5). Hence, it allowed for the reduction of counts (and then significantly the acquisition time), which kept the noise level the same as that of the $\mathrm{mCT}$ yet still improved contrast recovery. This reduction of acquisition time can be translated to a reduction of injected activity given the linear behavior of count with activity concentration when considering clinical activity range (supplementary material 4). A larger image matrix size $\left(440 \times 440,1.65 \times 1.65 \times 1.65 \mathrm{~mm}^{3}\right.$ voxel size $)$ could routinely be used while keeping the noise level compatible in order to benefit from a high 3D contrast recovery and hence a better detectability. It should be also emphasized that there was an almost strict equivalence in terms of noise between the mCT (three iterations, 21 subsets, 200 $\times 200$ ) and the Vision (five iterations, five subsets, $440 \times 440$ ) regardless of the number of net trues for the count rate used in this work (Fig. 5). This last finding highlights the possibility to maximize the detectability by using more iterations and the smallest voxel size while keeping the same noise level. It is worth noting that the approach developed in this study could also be conducted using post-filtering, as is done in routine clinical conditions. However, post-filtering was not applied in this study. Adding an extraparameter (post-filtering) would have made the analysis more complex (more possibilities to be taken into account) without adding significant valuable information.

The results obtained using the NEMA phantom paved the way to a new era for oncological exams. Apart from the reduction factor using the $440 \times 440$ matrix size presented above, it is possible to reach a factor of three (Table 5) using the $220 \times 220$ matrix size and still gain in contrast recovery if users are more interested in reducing injected activity or in speeding up the acquisition time for pediatric studies, for example. In other words, while a larger pixel size $\left(4 \times 4 \times 2 \mathrm{~mm}^{3}\right.$ for $\left.200 \times 200\right)$ had to be chosen for the $\mathrm{mCT}$ as a clinical standard to obtain a low noise level, with Vision 450 , a full resolution $\left(1.65 \times 1.65 \times 1.65 \mathrm{~mm}^{3}\right.$ for $\left.440 \times 440\right)$ could be used because of 
the noise reduction due to better TOF performance. Of note, these findings are roughly in line with those recently reported by Gnesin and colleagues [16]. They found a reduction factor of 3.12 when comparing $\mathrm{mCT}(512 \times 512$, three iterations, 21 subsets, and no post-filtering) to the Vision $600(512 \times 512$, four iterations, five subsets, and no post-filtering) for a coefficient of variation of $15 \%$. In the same settings (apart from the mCT matrix size used in this study $400 \times 400$ ), a reduction factor of 2.88 was reported for an IR of $16.5 \%$.

Obviously, this conclusion cannot be seen as a general rule valid for any type of patient, since only one phantom with specific geometrical properties was used. In that respect, the reduction factor may be different for heavier patients, who are more likely to benefit from the improved time resolution [17]. It is also worth noting that the reduction factors obtained in this work are only valid for the specific count rate for which they were calculated. The expectable dependency with count rate could be derived through the use of NECR curves which are available for both system. Additionally, while IR could be seen as a surrogate of noise and visual perception, the image texture may not be exactly the same when comparing two images with different voxel size (Fig. 6) although the IR is identical. Yet, more studies need to be conducted to consider different types of geometry using phantom or clinical data. The difficulty of using the same clinical data extracted from two different systems can be partially overcome with a random dual-imaging protocol as performed recently for the Vision 600 [18]. It is also possible to focus only on lesion detectability. A few interesting results were recently highlighted when comparing $\mathrm{mCT}$ and Vision 600 using a dedicated torso phantom [19]. That study found a possible reduction factor of between four and six for a nearly matched voxel size. Yet, optimization in this study was done on ${ }^{18} \mathrm{~F}$ and with count statistics typical of ${ }^{18} \mathrm{~F}$-FDG oncology imaging. Such optimization might need to be repeated for tracers that exhibit different statistics and contrast or organ distribution.

\section{Conclusion}

The Vision 450 exhibited very similar results as the Vision 600 in terms of spatial resolution, time resolution, and co-registration accuracy. It was shown that a significant reduction factor $(\approx 2)$ for time acquisition could be achieved using approximately the same matrix size while significantly improving the contrast recovery. The use of a larger matrix was also routinely feasible at a same noise level as that of the $\mathrm{mCT}$, with a reduction factor of 1.34 and a marked improvement of contrast recovery.

\section{Supplementary information}

\section{Supplementary information accompanies this paper at https://doi.org/10.1186/s40658-020-00323-w.}

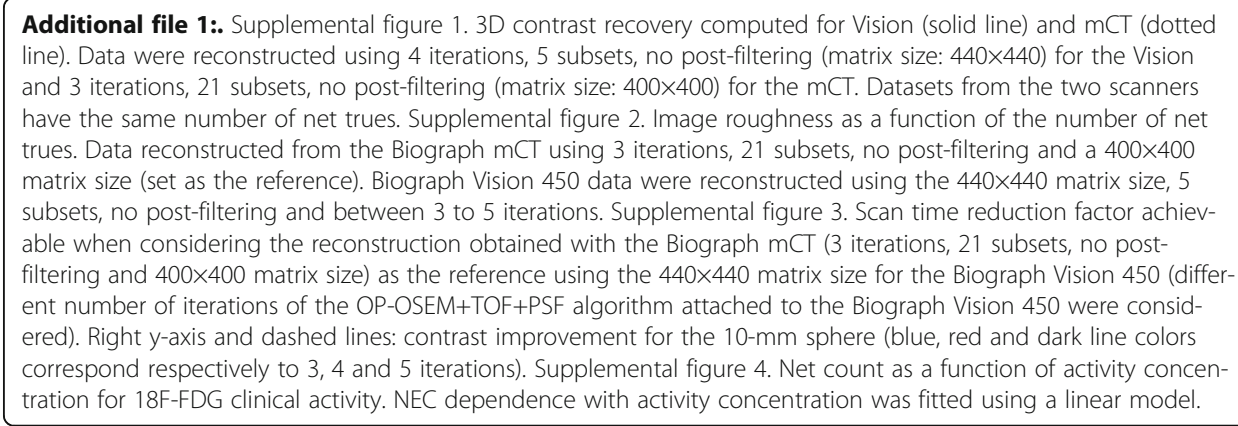

Additional file 1:. Supplemental figure 1. 3D contrast recovery computed for Vision (solid line) and $\mathrm{mCT}$ (dotted line). Data were reconstructed using 4 iterations, 5 subsets, no post-filtering (matrix size: $440 \times 440$ ) for the Vision and 3 iterations, 21 subsets, no post-filtering (matrix size: $400 \times 400$ ) for the $\mathrm{mCT}$. Datasets from the two scanners have the same number of net trues. Supplemental figure 2 . Image roughness as a function of the number of net trues. Data reconstructed from the Biograph $\mathrm{mCT}$ using 3 iterations, 21 subsets, no post-filtering and a $400 \times 400$ matrix size (set as the reference). Biograph Vision 450 data were reconstructed using the $440 \times 440$ matrix size, 5 subsets, no post-filtering and between 3 to 5 iterations. Supplemental figure 3. Scan time reduction factor achievable when considering the reconstruction obtained with the Biograph $\mathrm{mCT}$ (3 iterations, 21 subsets, no postfiltering and $400 \times 400$ matrix size) as the reference using the $440 \times 440$ matrix size for the Biograph Vision 450 (different number of iterations of the OP-OSEM+TOF+PSF algorithm attached to the Biograph Vision 450 were considered). Right $y$-axis and dashed lines: contrast improvement for the 10-mm sphere (blue, red and dark line colors correspond respectively to 3,4 and 5 iterations). Supplemental figure 4 . Net count as a function of activity concentration for 18F-FDG clinical activity. NEC dependence with activity concentration was fitted using a linear model. 


\section{Acknowledgements}

We thank Dr Bailly and Dr Barbaud from the Nuclear Medicine Department of the University Hospital, Nantes, for their help and support.

\section{Authors' contributions}

$T C, M C$, and BB designed the study. TC and LF contributed to data acquisition. TC, MC, and BB and YB contributed to the data analysis and interpretation. TC, MC, BB, CBM, CR, and FKB contributed to drafting of the manuscript together providing key input to the structure of the paper. All authors revised critically the manuscript and approved the final version.

\section{Funding}

This study has been supported in part by the French National Agency for Research called "Investissements d'Avenir" IRON Labex nº ANR-11-LABX-0018-01 and INCa-DGOS-Inserm_12558 (SIRIC ILIAD).

\section{Availability of data and materials}

The datasets generated during and/or analyzed during the current study are available from the corresponding author upon reasonable request.

\section{Ethics approval and consent to participate}

Ethical approval was waived by the local Ethics Committee of the University Hospital of Nantes in view of the retrospective nature of the study, and all procedures being performed were part of the routine care. Informed consent was obtained from all individual participants included in the study.

\section{Consent for publication}

The authors affirm that human research participants provided informed consent for publication of the related images.

\section{Competing interests}

Dr Maurizio Conti, Dr Bernard Bendriem, and Dr Yanic Bercier are Siemens employees. No other potential conflicts of interest relevant to this article exist.

\section{Author details}

${ }^{1}$ Nuclear Medicine Department, University Hospital of Nantes, Place Alexis Ricordeau, Nantes, France. ${ }^{2}$ Université de Nantes, CNRS, INSERM, CRCINA, Nantes, France. ${ }^{3}$ Nuclear Medicine Department, ICO, Boulevard Jacques Monod, Saint-Herblain, France. ${ }^{4}$ Siemens Medical Solutions USA, Inc., Knoxville, TN, USA.

Received: 21 May 2020 Accepted: 16 August 2020

Published online: 03 September 2020

\section{References}

1. Roncali E, Cherry SR. Application of silicon photomultipliers to positron emission tomography. Ann Biomed Eng. 2011; 39:1358-77.

2. Delso G, Fürst S, Jakoby B, Ladebeck R, Ganter C, Nekolla SG, et al. Performance measurements of the Siemens mMR integrated whole-body PET/MR scanner. J Nucl Med. 2011;52:1914-22.

3. Bisogni MG, Del Guerra A, Belcari N. Medical applications of silicon photomultipliers. Nucl Instr Meth Physics Research A 2019. 2019;926:118-28.

4. Levin CS, Maramraju SH, Khalighi MM, Deller TW, Delso G, Jansen F. Design features and mutual compatibility studies of the time-of-flight PET capable GE SIGNA PET/MR system. IEEE Trans Med Imaging. 2016;35:1907-14.

5. Conti M. Focus on time-of-flight PET: the benefits of improved time resolution. Eur J Nucl Med Mol Imaging. 2011;38: 1147-57.

6. Conti M. Why is TOF PET reconstruction a more robust method in the presence of inconsistent data? Phys Med Biol. 2011;56:155-68.

7. Conti M, Eriksson E, Westerwoudt V. Estimating image quality for future generations of TOF PET scanners. IEEE Trans Nucl Sci. 2013;60:87-94.

8. National Electrical Manufacturers Association Performance measurements of positron emission tomographs. Rosslyn, VA: National Electrical Manufacturers Association; 2018. NEMA Standards Publication NU 2-2018.

9. van Sluis J, de Jong J, Schaar J, Noordzij W, van Snick P, Dierckx R, et al. Performance characteristics of the digital biograph vision PET/CT system. J Nucl Med. 2019;60:1031-6.

10. Wang GC, Li X, Niu X, Du H, Balakrishnan K, Ye H, et al. PET timing performance measurement method using NEMA NEC phantom. IEEE Trans Nucl Sci. 2016;63:1335-42.

11. Jakoby BW, Bercier Y, Conti M, Casey ME, Bendriem B, Townsend DW. Physical and clinical performance of the $m C T$ time-of-flight PET/CT scanner. Phys Med Biol. 2011;56:2375-89.

12. Tong S, Alessio AM, Kinahan PE. Noise and signal properties in PSF-based fully 3D PET image reconstruction: an experimental evaluation. Phys Med Biol. 2010;55:1453-73.

13. Kunnen B, Beijst C, Lam MGEH, Viergever MA, de Jong HWAM. Comparison of the biograph vision and biograph mCT for quantitative 90Y PET/CT imaging for radioembolisation. EJNMMI Phys. 2020;7:14.

14. Carlier T, Ferrer L, Necib H, Bodet-Milin C, Rousseau R, Kraeber-Bodéré F. Clinical NECR in 18F-FDG PET scans: optimization of injected activity and variable acquisition time. Relationship with SNR. Phys Med Biol. 2014;59:6417-30.

15. Eriksson L, Townsend D, Conti M, Eriksson M, Rothfuss H, Schmand M, et al. An investigation of sensitivity limits in PET scanners. Nucl Instr Meth Physics Research A. 2007;580:836-42.

16. Gnesin S, Kieffer C, Zeimpekis K, Papazyan JP, Guignard R, Prior JO, et al. Phantom-based image quality assessment of clinical ${ }^{18}$ F-FDG protocols in digital PET/CT and comparison to conventional PMT-based PET/CT. EJNMMI Phys. 2020;7:1. 
17. El Fakhri G, Surti S, Trott CM, Scheuermann J, Karp JS. Improvement in lesion detection with whole-body oncologic time-of-flight PET. J Nucl Med. 2011;52:347-53.

18. van Sluis J, Boellaard R, Somasundaram A, van Snick PH, Borra RJH, Dierckx RAJO, et al. Image quality and semiquantitative measurements on the biograph vision PET/CT system: initial experiences and comparison with the biograph mCT. J Nucl Med. 2020;61:129-35.

19. Surti S, Viswanath V, Daube-Witherspoon ME, Conti M, Casey ME, Karp JS. Benefit of improved performance with stateof-the art digital PET/CT for lesion detection in oncology. J Nucl Med. 2020; [ahead of print].

\section{Publisher's Note}

Springer Nature remains neutral with regard to jurisdictional claims in published maps and institutional affiliations.

Submit your manuscript to a SpringerOpen ${ }^{\odot}$ journal and benefit from:

- Convenient online submission

- Rigorous peer review

- Open access: articles freely available online

- High visibility within the field

- Retaining the copyright to your article 